\title{
An elementary approach to the location of the maximum Stirling number(s) of the second kind
}

\author{
Horst Wegner \\ Horst Wegner studierte Mathematik an der Universität Hamburg. Nach dem Diplom \\ 1966 und kurzer Tätigkeit in der Industrie promovierte er 1970 über ein Problem zu \\ Stirlingschen Zahlen zweiter Art an der Universität Köln. Seit 1973 ist er als Akade- \\ mischer Oberrat an der Universität Duisburg tätig, zunächst in der Lehrerausbildung \\ und seit 1982 im Fachgebiet Stochastik.
}

\section{The unimodality}

The number of ways of partitioning a set of $n$ elements into $k$ nonempty subsets is usually denoted $S(n, k)$. These numbers are called Stirling numbers of the second kind, which were so named by Niels Nielsen [5], who wrote in German "Stirlingsche Zahlen".

Obviously $S(n, 1)=S(n, n)=1$ and $S(n, k)=0$ for all $k>n$. Moreover it is useful to put $S(0,0)=1$ and $S(0, k)=S(n, 0)=0$ for all $n, k \in \mathbb{N}$. Elementary combinatorial arguments lead us to the two following recurrences

$$
\begin{aligned}
& S(n+1, k)=k S(n, k)+S(n, k-1), \quad n, k \in \mathbb{N}, \\
& S(n+1, k)=\sum_{i=0}^{n}\left(\begin{array}{l}
n \\
i
\end{array}\right) S(i, k-1), \quad n, k \in \mathbb{N} .
\end{aligned}
$$

Auf wie viele Weisen lässt sich eine Menge von $n$ Elementen in $k$ nicht-leere Teilmengen zerlegen? Die Antwort hierauf liefern die Stirlingschen Zahlen $S(n, k)$ zweiter Art, wobei der Zusatz zweiter Art historische Gründe hat. Betrachtet man für fest gewähltes $n$ die Abhängigkeit der $S(n, k)$ von $k$, stellt man fest, dass die $S(n, k)$ zunächst zunehmen, ein Maximum erreichen und dann wieder abnehmen. Dabei kann bisher nicht ausgeschlossen werden, dass das Maximum für zwei benachbarte $k$ angenommen wird. Die Frage, wo die $S(n, k)$ ihr Maximum annehmen, wurde von verschiedenen Autoren mittels asymptotischer Aussagen behandelt. In der vorliegenden Arbeit werden mit elementaren Mitteln exakte Aussagen über die Lage des Maximums gewonnen. 
Using (1.1) and (1.2), it can be shown by mathematical induction on the value of $n$ that for fixed $n$ the numbers $S(n, k)$ are unimodal in the following sense (see [1], [6]).

Theorem 1.1. For all $n \in \mathbb{N}$ there exists $a K_{n} \in \mathbb{N}, 1 \leq K_{n} \leq n$, such that

$$
\begin{array}{lll}
S(n, k-1)<S(n, k) & \text { for } & 1 \leq k \leq K_{n}-1, \\
S(n, k-1) \leq S(n, k) & \text { for } & k=K_{n}, \\
S(n, k-1)>S(n, k) & \text { for } & K_{n}+1 \leq k \leq n+1 .
\end{array}
$$

Furthermore $0 \leq K_{n+1}-K_{n} \leq 1$.

If we do without the inequality $K_{n+1} \leq K_{n}+1$, Theorem 1.1 can be proved using only (1.1) (see [3]).

With regard to our further investigations it is useful to define

$$
K_{n}^{*}:=\left\{\begin{array}{lll}
K_{n}-1 & \text { if } \quad S\left(n, K_{n}-1\right)=S\left(n, K_{n}\right), \\
K_{n} & \text { if } \quad S\left(n, K_{n}-1\right)<S\left(n, K_{n}\right) .
\end{array}\right.
$$

According to (1.3), the proof by induction of Theorem 1.1 shows that more precisely

$$
K_{n} \leq K_{n+1}^{*} \leq K_{n+1} \leq K_{n}+1 .
$$

Clearly $K_{2}^{*}=K_{2}-1$. It is not known to the author whether there is another case such that $K_{n}^{*}=K_{n}-1$. It seems to be an unsolved problem whether $S(n, k)$ always has a single maximum for $n \geq 3$. Some results concerning this problem and the value $K_{n}$ have been established (see e.g. [2], [3], [4], [7]).

The first aim of this paper is to obtain bounds for $K_{n}, K_{n}^{*}$, using quite elementary methods. The results, attained in this way, will be stated in the Theorems 3.2 and 3.5. Finally, in the last section, we will show how to determine exact values of $K_{n}, K_{n}^{*}$.

\section{Preparatory remarks}

Let $n, k \in \mathbb{N}$ and let $X, Y$ be sets with $|X|=n,|Y|=k$. It is evident that $k ! S(n, k)$ is the number of surjective functions from $X$ to $Y$. Thus, by simple combinatorial considerations, we obtain for the number of all functions from $X$ to $Y$ the following formula:

$$
k^{n}=\sum_{i=0}^{k-1}\left(\begin{array}{c}
k \\
k-i
\end{array}\right)(k-i) ! S(n, k-i) .
$$

Hence

$$
\frac{k^{n}}{k !}=\sum_{i=0}^{k-1} \frac{1}{i !} S(n, k-i)
$$

Furthermore, using the principle of inclusion and exclusion, the number of non-surjective functions from $X$ to $Y$ is

$$
k^{n}-k ! S(n, k)=\sum_{i=1}^{k-1}(-1)^{i-1}\left(\begin{array}{c}
k \\
k-i
\end{array}\right)(k-i)^{n}
$$


(see [8], Section 4), and this implies

$$
S(n, k)=\sum_{i=0}^{k-1} \frac{(-1)^{i}}{i !} \cdot \frac{(k-i)^{n}}{(k-i) !}
$$

By the formulas (2.1), (2.2) (clearly, they are well-known), we obtain

$$
\frac{k^{n}}{k !}-\frac{(k-1)^{n}}{(k-1) !}=\sum_{i=0}^{k-1} \frac{1}{i !}(S(n, k-i)-S(n, k-i-1))
$$

and

$$
S(n, k)-S(n, k-1)=\sum_{i=0}^{k-1} \frac{(-1)^{i}}{i !}\left(\frac{(k-i)^{n}}{(k-i) !}-\frac{(k-i-1)^{n}}{(k-i-1) !}\right) .
$$

The last two formulas will lead us to the desired results in Theorem 3.2 and Theorem 3.5.

\section{Bounds for $K_{n}, K_{n}^{*}$}

First we want to establish an upper bound for $K_{n}$, which is already given in [7]. Previously we are beginning with a result, which is evident.

Lemma 3.1. Let $n \in \mathbb{N}, n \geq 2$. Then there is a unique $s_{n} \in(2, \infty)$ such that

$$
x\left(1-\frac{1}{x}\right)^{n}\left\{\begin{array}{lll}
<1 & \text { for } & 2 \leq x<s_{n}, \\
=1 & \text { for } & x=s_{n} \\
>1 & \text { for } & x>s_{n} .
\end{array}\right.
$$

Theorem 3.2. Let $n \in \mathbb{N}, n \geq 2$, and let $K_{n}$ be given by Theorem 1.1. Furthermore let $s_{n}$ be the unique root of $x\left(1-\frac{1}{x}\right)^{n}=1$ in the interval $(2, \infty)$ (see Lemma 3.1). Then $K_{n} \leq\left[s_{n}\right]$.

Proof. Let $k=K_{n}$. Then, by (2.3),

$$
\frac{k^{n-1}}{(k-1) !}\left(1-k\left(1-\frac{1}{k}\right)^{n}\right)=\frac{k^{n}}{k !}-\frac{(k-1)^{n}}{(k-1) !} \geq 0 .
$$

Hence $k\left(1-\frac{1}{k}\right)^{n} \leq 1$, and then Lemma 3.1 implies $K_{n}=k \leq s_{n}$. Hence $K_{n} \leq\left[s_{n}\right]$.

Before giving a lower bound for $K_{n}^{*}$ we establish two further lemmas.

Lemma 3.3. Let $n \in \mathbb{N}$. Then the function

$$
f(x):=\frac{1}{x}\left(1+\frac{1}{x}\right)^{n-1}+x\left(1-\frac{1}{x}\right)^{n}
$$

is strictly convex on the interval $[1, \infty)$.

Proof. It is easy to show that $f^{\prime \prime}(x)>0$ for all $x \geq 1$. 
Lemma 3.4. Let $n \in \mathbb{N}, n \geq 3$, and let $s_{n}$ be given by Lemma 3.1. Then there is a unique $r_{n} \in\left(2, s_{n}\right)$ such that

$$
\frac{1}{x-1}\left(1+\frac{1}{x-1}\right)^{n-1}+(x-1)\left(1-\frac{1}{x-1}\right)^{n}\left\{\begin{array}{lll}
>2 & \text { for } & 2 \leq x<r_{n} \\
=2 & \text { for } & x=r_{n} \\
<2 & \text { for } & r_{n}<x \leq s_{n}
\end{array}\right.
$$

Proof. For abbreviation we put

$$
g(x):=\frac{1}{x-1}\left(1+\frac{1}{x-1}\right)^{n-1}+(x-1)\left(1-\frac{1}{x-1}\right)^{n} .
$$

Obviously

(i) $g(2)>2$.

Now let $x=s_{n}$. Then Lemma 3.1 implies

$$
\begin{aligned}
& 1=x\left(1-\frac{1}{x}\right)^{n}>(x-1)\left(1-\frac{1}{x-1}\right)^{n} \text { and } \\
& 1=\frac{1}{x}\left(1-\frac{1}{x}\right)^{-n}=\frac{1}{x-1}\left(1+\frac{1}{x-1}\right)^{n-1} .
\end{aligned}
$$

These two relations imply

(ii) $g\left(s_{n}\right)<2$.

With the function $f$ given in Lemma 3.3 we have $g(x)=f(x-1)$, and then it follows from Lemma 3.3 that $g$ ist strictly convex on the interval $[2, \infty)$. With regard to (i), (ii), this implies that there is a unique $r_{n} \in\left(2, s_{n}\right)$ such that

$$
g(x)\left\{\begin{array}{lll}
>2 & \text { for } \quad 2 \leq x<r_{n} \\
=2 & \text { for } \quad x=r_{n} \\
<2 & \text { for } \quad r_{n}<x \leq s_{n}
\end{array}\right.
$$

Now we are prepared to establish a lower bound for $K_{n}^{*}$.

Theorem 3.5. Let $n \in \mathbb{N}, n \geq 3$, and let $K_{n}^{*}$ be given by Theorem 1.1 and (1.3). Furthermore let $r_{n}$ be the unique root of

$$
\frac{1}{x-1}\left(1+\frac{1}{x-1}\right)^{n-1}+(x-1)\left(1-\frac{1}{x-1}\right)^{n}=2
$$

in the interval $\left(2, s_{n}\right)$ (see Lemma 3.4), where $s_{n}$ is given by Lemma 3.1. Then $\left[r_{n}\right] \leq K_{n}^{*}$.

Proof. Our aim is to apply (2.4).

Let $k=\left[r_{n}\right]$, hence $k \geq 2$. Then, for $i=0,1, \ldots, k-1$ we put

$$
\Delta_{i}:=\frac{(k-i)^{n}}{(k-i) !}-\frac{(k-i-1)^{n}}{(k-i-1) !}=\frac{(k-i)^{n}}{(k-i) !}\left(1-(k-i)\left(1-\frac{1}{k-i}\right)^{n}\right) .
$$


Since $1 \leq k-i \leq r_{n}<s_{n}$, it follows from Lemma 3.1 that

$$
\Delta_{i}>0 \text { for } i=0,1, \ldots, k-1 \text {. }
$$

Moreover, for $i=0,1, \ldots, k-2$ one has

$$
\begin{aligned}
\Delta_{i}-\Delta_{i+1}= & \frac{1}{(k-i-1) !}\left((k-i)^{n-1}-2(k-i-1)^{n}+(k-i-1)(k-i-2)^{n}\right) \\
=\frac{(k-i-1)^{n}}{(k-i-1) !}( & \frac{1}{k-i-1}\left(1+\frac{1}{k-i-1}\right)^{n-1} \\
& \left.\quad+(k-i-1)\left(1-\frac{1}{k-i-1}\right)^{n}-2\right)
\end{aligned}
$$

Since $2 \leq k-i \leq r_{n}$, it follows then from Lemma 3.4 that

$$
\Delta_{i}-\Delta_{i+1} \geq 0 \text { for } i=0,1, \ldots, k-2
$$

In particular, we obtain for $i=k-2$

$$
\Delta_{k-2}-\Delta_{k-1}=2^{n-1}-2 \geq 2^{2}-2>0 .
$$

With respect to (3.1), we obtain from (3.2), (3.3)

$$
\frac{\Delta_{i}}{i !} \geq \frac{\Delta_{i+1}}{(i+1) !} \text { for } i=0,1, \ldots, k-2
$$

and in particular

$$
\frac{\Delta_{k-2}}{(k-2) !}>\frac{\Delta_{k-1}}{(k-1) !}
$$

According to (3.1), (3.4), (3.5), it follows from (2.4) that

$$
S(n, k)-S(n, k-1)=\sum_{i=0}^{k-1}(-1)^{i} \frac{\Delta_{i}}{i !}>0 .
$$

Hence $K_{n}^{*} \geq k=\left[r_{n}\right]$.

This section shall end with some examples illustrating the results of Theorems 3.2 and 3.5:

\begin{tabular}{c|rrrrrrrrrr}
$n$ & 10 & 25 & 50 & 100 & 250 & 500 & 1000 & 2500 & 5000 & 10000 \\
\hline$\left[s_{n}\right]$ & 6 & 10 & 17 & 29 & 61 & 107 & 190 & 415 & 755 & 1383 \\
{$\left[r_{n}\right]$} & 5 & 9 & 16 & 27 & 58 & 103 & 185 & 407 & 745 & 1370
\end{tabular}




\section{Exact values of $\boldsymbol{K}_{\boldsymbol{n}}$}

In many cases the exact values of $K_{n}, K_{n}^{*}$ can be determined by a procedure first published by Comtet and Fiolet [4], which shall be described here.

We know that the partial sums of (2.2) successively overcount and undercount the number $S(n, k)$ (see [8]). Thus, for $l=0,1, \ldots, k-1$ the expressions

$$
\begin{aligned}
& \sum_{i=0}^{l} \frac{(-1)^{i}}{i !} \cdot \frac{(k-i)^{n}}{(k-i) !}-\sum_{i=0}^{l-1} \frac{(-1)^{i}}{i !} \cdot \frac{(k-1-i)^{n}}{(k-1-i) !} \\
& =\sum_{i=0}^{l}(-1)^{i} \frac{i+1}{i !} \cdot \frac{(k-i)^{n}}{(k-i) !}=\frac{k^{n}}{k !} \sum_{i=0}^{l}(-1)^{i}(i+1)\left(\begin{array}{c}
k \\
i
\end{array}\right)\left(1-\frac{i}{k}\right)^{n}
\end{aligned}
$$

are successively upper and lower bounds for the difference $S(n, k)-S(n, k-1)$. Putting for abbreviation

$$
v(n, k, l):=\sum_{i=0}^{l}(-1)^{i}(i+1)\left(\begin{array}{l}
k \\
i
\end{array}\right)\left(1-\frac{i}{k}\right)^{n},
$$

we have for $l=0,1, \ldots, k-1$ the relations

$$
S(n, k)-S(n, k-1) \begin{cases}\leq \frac{k^{n}}{k !} v(n, k, l), & \text { if } l \text { is even, } \\ \geq \frac{k^{n}}{k !} v(n, k, l), & \text { if } l \text { is odd. }\end{cases}
$$

Using (4.1), Theorem 1.1, (1.3), we obtain

$$
\left\{\begin{array}{c}
l \text { even } \wedge v(n, k, l)<0 \Rightarrow K_{n} \leq k-1 \\
l \text { odd } \wedge v(n, k, l)>0 \Rightarrow K_{n}^{*} \geq k
\end{array}\right.
$$

Now let us apply (4.2) to our last example of Section 3, which gives us

$$
1370 \leq K_{10000}^{*} \leq K_{10000} \leq 1383
$$

As a first step we check $v(10000, k, l)$ for $k=1383$. Then $l=6$ is the first even number such that $v(10000,1383, l)$ is negative, namely

$$
v(10000,1383,6)=-0.000510 \ldots
$$

Thus, by (4.2), $K_{10000} \leq 1382$. Already a second step shows us that

$$
v(10000,1382,7)=0.000314 \ldots>0
$$

and therefore (4.2) implies $K_{10000}^{*} \geq 1382$.

Hence $K_{10000}=K_{10000}^{*}=1382$. 
This example shows us that the upper bound $\left[s_{n}\right]$ from Theorem 3.2 is much sharper than the lower bound from Theorem 3.5. This fact will be emphasized by many other examples. (According to (1.4), (4.2), it is quite easy to determine $K_{n+1}$, if the value of $K_{n}$ is given.)

In the overwhelming majority of cases we obtain $K_{n}=\left[s_{n}\right]-1$ and only in a few cases $K_{n}=\left[s_{n}\right]$. So we can fall into temptation to suppose that $K_{n} \geq\left[s_{n}\right]-1$ for all $n \geq 3$. But no elementary proof of this inequality can be offered.

\section{References}

[1] Aigner, M.: Combinatorial Theory. Springer, Berlin 1997.

[2] Canfield, E.R.: On the location of the maximum Stirling number(s) of the second kind. Studies Appl. Math. 59 (1978), 83-93.

[3] Canfield, E.R.; Pomerance, C.: On the problem of uniqueness for the maximum Stirling number(s) of the second kind. Integers 2, Paper A01, electronic only (2002), 13 pp.

[4] Comtet, L.; Fiolet, M.: Sur le mode de nombres de Stirling de seconde espèce. C.R. Acad. Sci. Paris, Sér. A 280 (1975), 1263-1265.

[5] Nielsen, N.: Handbuch der Theorie der Gammafunktion. Teubner, Leipzig 1906.

[6] Rennie, B.C.; Dobson, A.J.: On Stirling numbers of the second kind. J. Comb. Theory 7 (1969), 116-121.

[7] Wegner, H.: Über das Maximum bei Stirlingschen Zahlen zweiter Art. J. Reine Angew. Math. 262/263 (1973), 134-143.

[8] Wegner, H.: Stirling numbers of the second kind and Bonferroni's inequalities. Elem. Math. 60 (2005), 124-129.

Horst Wegner

Universität Duisburg-Essen

Campus Duisburg

Fachbereich Mathematik

Forsthausweg 2

D-47057 Duisburg, Deutschland

e-mail: wegner@math.uni-duisburg.de 